\title{
A regularization algorithm for a common solution of generalized equilibrium problem, fixed point problem and the zero points of the sum of two operators
}

Ming $\operatorname{Tian}^{1,2^{*}}$ and Si-Wen Jiao'

\author{
"Correspondence: \\ tianming1963@126.com \\ ${ }^{1}$ College of Science, Civil Aviation \\ University of China, Tianjin, 300300, \\ China \\ ${ }^{2}$ Tianjin Key Laboratory for \\ Advanced Signal Processing, Civil \\ Aviation University of China, Tianjin, \\ 300300, China
}

\begin{abstract}
For finding a common solution of generalized equilibrium problem, fixed point problem and the zero points of the sum of two operators, a regularization algorithm is established in the framework of real Hilbert spaces. And the strong convergence theorem is obtained under certain assumptions. The main results presented in this paper are useful in nonlinear analysis and optimization. Moreover, the results and corollaries extend the corresponding conclusions proposed by many authors.

MSC: 58E35; 47H09; 65J15

Keywords: iterative method; fixed point; generalized equilibrium problem; infinitely nonexpansive mappings; maximal monotone operator; resolvent; inverse-strongly monotone mapping; strict pseudo-contraction; variational inequality
\end{abstract}

\section{Introduction}

In this paper, assume that $H$ is a real Hilbert space with the inner product $\langle\cdot, \cdot \cdot\rangle$ and the norm $\|\cdot\|$, and let $C$ be a nonempty, closed and convex subset of $H$. Let $\mathbb{N}$ and $\mathbb{R}$ be the sets of positive integers and real numbers, respectively. In the following, we recall some mappings which will often be used in this paper.

- $f: C \rightarrow C$ is said to be $k$-contractive iff there exists a constant $k \in(0,1)$ such that $\|f(x)-f(y)\| \leq k\|x-y\|$ for all $x, y \in C$.

- $S: C \rightarrow C$ is said to be nonexpansive iff $\|S x-S y\| \leq\|x-y\|$ for all $x, y \in C$.

- $T: H \rightarrow H$ is said to be firmly nonexpansive iff $\|T x-T y\|^{2} \leq\langle T x-T y, x-y\rangle$ for all $x, y \in H$.

- $P_{C}: H \rightarrow C$ is said to be metric projection iff $\left\|x-P_{C} x\right\| \leq\|x-y\|$ for all $x \in H$ and $y \in C$

- $A: H \rightarrow H$ is said to be monotone iff $\langle x-y, A x-A y\rangle \geq 0$ for all $x, y \in H$.

- Given a number $\eta>0, A: H \rightarrow H$ is said to be $\eta$-strongly monotone iff $\langle x-y, A x-A y\rangle \geq \eta\|x-y\|^{2}$ for all $x, y \in H$.

- Given a number $\alpha>0, A: C \rightarrow H$ is said to be $\alpha$-inverse-strongly monotone ( $\alpha$-ism) iff $\langle x-y, A x-A y\rangle \geq \alpha\|A x-A y\|^{2}$ for all $x, y \in C$.

(c) 2015 Tian and Jiao. This article is distributed under the terms of the Creative Commons Attribution 4.0 International License (http://creativecommons.org/licenses/by/4.0/), which permits unrestricted use, distribution, and reproduction in any medium, provided you give appropriate credit to the original author(s) and the source, provide a link to the Creative Commons license, and indicate if changes were made. 
- $Y: C \rightarrow H$ is a strict pseudo-contraction [1] if there exists $t \in \mathbb{R}$ with $0 \leq t<1$ such that $\|Y x-Y y\|^{2} \leq\|x-y\|^{2}+t\|(I-Y) x-(I-Y) y\|^{2}$ for all $x, y \in C$.

First, we introduce the following generalized equilibrium problem.

Find $x \in C$ such that

$$
F(x, y)+\langle T x, y-x\rangle \geq 0, \quad \forall y \in C,
$$

where $T: C \rightarrow H$ is a monotone mapping and $F: C \times C \rightarrow \mathbb{R}$ is a bifunction.

In this paper, we use $\operatorname{GEP}(F, T)$ to denote the set of such $x \in C$, i.e., $\operatorname{GEP}(F, T)=\{x \in C$ : $F(x, y)+\langle T x, y-x\rangle \geq 0, \forall y \in C\}$.

In the case of $T \equiv 0$, problem (1.1) is reduced to the following equilibrium problem [2].

Find $x \in C$ such that

$$
F(x, y) \geq 0, \quad \forall y \in C
$$

In this paper, we use $\operatorname{EP}(F)$ to denote the set of such $x \in C$, i.e., $\operatorname{EP}(F)=\{x \in C: F(x, y) \geq$ $0, \forall y \in C\}$.

In the case of $F \equiv 0$, problem (1.1) is reduced to the classical variational inequality.

To study equilibrium problems (1.1) and (1.2), we may assume that $F$ satisfies the following conditions:

(A1) $F(x, x)=0$ for all $x \in C$;

(A2) $F$ is monotone, i.e., $F(x, y)+F(y, x) \leq 0$ for all $x, y \in C$;

(A3) for each $x, y, z \in C$,

$$
\lim _{t \downarrow 0} \sup F(t z+(1-t) x, y) \leq F(x, y)
$$

(A4) for each $x \in C, y \mapsto F(x, y)$ is convex and weakly lower semicontinuous.

Many problems can be transformed into finding solutions of equilibrium problems (1.1) and (1.2), for instance, image recovery, network allocation, inverse problems, transportation problems and optimization problems; see [2-7] and the references therein. Recently, many regularization methods have been extensively studied for solving solutions of equilibrium problems (1.1) and (1.2); see [7-13] and the references therein.

Second, we introduce the following fixed point problem for a family of infinitely nonexpansive mappings. Consider the fixed point problem

$$
\operatorname{Fix}(S):=\{x \in C: x=S x\},
$$

where $S: C \rightarrow C$ is a mapping, we use Fix $(S)$ to denote the fixed point set of $S$. If $C$ is a bounded, closed and convex subset of $H$, then $\operatorname{Fix}(S)$ is not empty; see [1].

Let $\left\{S_{i}: C \rightarrow C\right\}$ be a family of infinitely nonexpansive mappings and $\left\{\gamma_{i}\right\}$ be a nonnegative real sequence with $0 \leq \gamma_{i}<1, \forall i \geq 1$. For $n \geq 1$, define a mapping $W_{n}: C \rightarrow C$ as follows:

$$
\begin{aligned}
& U_{n, n+1}=I, \\
& U_{n, n}=\gamma_{n} S_{n} U_{n, n+1}+\left(1-\gamma_{n}\right) I,
\end{aligned}
$$




$$
\begin{aligned}
& U_{n, n-1}=\gamma_{n-1} S_{n-1} U_{n, n}+\left(1-\gamma_{n-1}\right) I, \\
& \ldots, \\
& U_{n, k}=\gamma_{k} S_{k} U_{n, k+1}+\left(1-\gamma_{k}\right) I, \\
& U_{n, k-1}=\gamma_{k-1} S_{k-1} U_{n, k}+\left(1-\gamma_{k-1}\right) I, \\
& \ldots, \\
& U_{n, 2}=\gamma_{2} S_{2} U_{n, 3}+\left(1-\gamma_{2}\right) I, \\
& W_{n}=U_{n, 1}=\gamma_{1} S_{1} U_{n, 2}+\left(1-\gamma_{1}\right) I .
\end{aligned}
$$

Such a mapping $W_{n}$ is nonexpansive from $C$ to $C$ and it is called a $W$-mapping generated by $S_{n}, S_{n-1}, \ldots, S_{1}$ and $\gamma_{n}, \gamma_{n-1}, \ldots, \gamma_{1}$; see [14] and the references therein.

Third, we introduce the problem of zero points of a maximal monotone mapping

$$
M^{-1} 0=\{x \in H: 0 \in M x\}
$$

where $M$ is a mapping of $H$ into $2^{H}$, the effective domain of $M$ is denoted by $\operatorname{dom} M$ or $D(M)$, that is, $\operatorname{dom} M=\{x \in H: M x \neq \emptyset\}$. A multi-valued mapping $M$ is said to be a monotone mapping on $H$ if $\langle x-y, u-v\rangle \geq 0$ for all $x, y \in \operatorname{dom} M, u \in M x, v \in M y$. A monotone mapping $M$ on $H$ is said to be maximal if its graph is not properly contained in the graph of any other monotone mapping on $H$. It is well known that a monotone mapping $M$ is maximal if and only if, for any $(x, u) \in H \times H,\langle x-y, u-v\rangle \geq 0$ for all $(y, v) \in \operatorname{Graph}(M)$ implies $u \in M x$.

For a maximal monotone mapping $M$ on $H$ and $r>0$, we may define a single-valued mapping $J_{r}=(I+r M)^{-1}: H \rightarrow \operatorname{dom} M$, which is called the resolvent of $M$ for $r$. It is easy to see that $M^{-1} 0=\operatorname{Fix}\left(J_{r}\right)$ for all $r>0$, and the resolvent $J_{r}$ is firmly nonexpansive, i.e.,

$$
\left\|J_{r} x-J_{r} y\right\|^{2} \leq\left\langle x-y, J_{r} x-J_{r} y\right\rangle, \quad \forall x, y \in H .
$$

Finally, we introduce the subdifferential of a lower semicontinuous convex function and an indicator function.

Let $h$ be a proper lower semicontinuous convex function on a Hilbert space $H$ into $(-\infty, \infty]$. Then the subdifferential $\partial h$ of $h$ is defined as follows:

$$
\partial h(x)=\{z \in H: h(x)+\langle z, y-x\rangle \leq h(y), \forall y \in H\}
$$

for all $x \in H$. From Rockafellar [15] we know that $\partial h$ is a maximal monotone operator. Let $i_{C}$ be the indicator function of $C$ ( $C$ is a nonempty closed convex subset of $H$ ), i.e.,

$$
i_{C}(x)= \begin{cases}0, & x \in C \\ \infty, & x \notin C\end{cases}
$$

Then $i_{C}$ is a proper lower semicontinuous convex function on $H$ and the subdifferential $\partial i_{C}$ of $i_{C}$ is a maximal monotone mapping. So we can define the resolvent $J_{r}$ of $\partial i_{C}$ for $r>0$, i.e.,

$$
J_{r} x=\left(I+r \partial i_{C}\right)^{-1} x
$$


for all $x \in H$. We have that for any $x \in H$ and $q \in C$,

$$
\begin{aligned}
q=J_{r} x & \Longleftrightarrow x \in q+r \partial i_{C}(q) \\
& \Longleftrightarrow x \in q+r N_{C}(q) \\
& \Longleftrightarrow x-q \in r N_{C}(q) \\
& \Longleftrightarrow \frac{1}{r}\langle x-q, p-q\rangle \leq 0, \quad \forall p \in C \\
& \Longleftrightarrow\langle x-q, p-q\rangle \leq 0, \quad \forall p \in C \\
& \Longleftrightarrow q=P_{C} x,
\end{aligned}
$$

where $N_{C}(q)$ is the normal cone to $C$ at $q$, i.e.,

$$
N_{C}(q)=\{z \in H:\langle z, p-q\rangle \leq 0, \forall p \in C\}
$$

In the present paper, we study the equilibrium problems (1.1) and (1.2), the fixed point problem for a family of infinitely nonexpansive mappings, and the problem of zero points of maximal monotone mapping (1.3). Motivated and inspired by the research going on in this direction, we propose a new regularization algorithm, and it is proved that the sequence generated by this algorithm converges strongly to a common solution of the above three problems. The results presented in this paper improve and extend the corresponding results in Chang et al. [7], Takahashi and Takahashi [8], Hao [16] and Yuan and Zhang [17].

The structure of this paper is set as follows. In Section 2, we introduce some lemmas which will be used in the proof of theorems. The main result, that is, the strong convergence of the regularization algorithm, is proved in Section 3. Corollaries to generalized equilibrium problem and the zero points of the sum of two operators are presented in Section 4. And the conclusion of this paper is given in the final section, i.e., Section 5.

\section{Preliminaries}

In the following, we give some useful lemmas, which will often be used in the proof of the main results and their corollaries.

Let $H$ be a real Hilbert space with the inner product $\langle\cdot, \cdot\rangle$ and the norm $\|\cdot\|$. When $\left\{x_{n}\right\}$ is a sequence in $H$, we define that the strong convergence of $\left\{x_{n}\right\}$ is a sequence in $H$, we denote the strong convergence of $\left\{x_{n}\right\}$ to $x \in H$ by $x_{n} \rightarrow x$ and the weak convergence by $x_{n} \rightarrow x$. Let $C$ be a nonempty closed convex subset of a Hilbert space $H$, and let $T$ : $C \rightarrow H$ be a mapping. We denote by $\operatorname{Fix}(T)$ the set of fixed points for $T$. If $T: C \rightarrow H$ is a nonexpansive mapping, then $\operatorname{Fix}(T)$ is closed and convex; see [18].

Firstly, we recall the metric (nearest point) projection from $H$ onto $C$ is the mapping $P_{C}: H \rightarrow C$ which is defined as follows: given $x \in H, P_{C} x$ is the unique point in $C$ with the property

$$
\left\|x-P_{C} x\right\|=\inf _{y \in C}\|x-y\|=: d(x, C)
$$

$P_{C}$ is characterized as follows. 
Lemma 2.1 Given $x \in H$ and $y \in C$. Then $y=P_{C} x$ if and only if the following inequality holds:

$$
\langle x-y, y-z\rangle \geq 0, \quad \forall z \in C
$$

Then we introduce the lemma below, which shows the uniqueness of solution of the variational inequality.

Lemma 2.2 [19] Let $H$ be a Hilbert space, $C$ be a closed convex subset of $H$, and $f: C \rightarrow C$ be a contraction with coefficient $\alpha<1$. Then

$$
\langle x-y,(I-f) x-(I-f) y\rangle \geq(1-\alpha)\|x-y\|^{2}, \quad x, y \in C .
$$

That is, $I-f$ is strongly monotone with coefficient $1-\alpha$.

Lemma 2.3 [2] Let $F: C \times C \rightarrow \mathbb{R}$ be a bifunction satisfying (A1)-(A4). Then, for any $r>0$ and $x \in H$, there exists $z \in C$ such that

$$
F(z, y)+\frac{1}{r}\langle y-z, z-x\rangle \geq 0, \quad \forall y \in C .
$$

Define a mapping $T_{r}: H \rightarrow C$ as follows:

$$
T_{r} x=\left\{z \in C: F(z, y)+\frac{1}{r}\langle y-z, z-x\rangle \geq 0, \forall y \in C\right\}, \quad x \in H,
$$

then the following conclusions hold:

(1) $T_{r}$ is single-valued;

(2) $T_{r}$ is firmly nonexpansive, i.e., for any $x, y \in H$,

$$
\left\|T_{r} x-T_{r} y\right\|^{2} \leq\left\langle T_{r} x-T_{r} y, x-y\right\rangle
$$

(3) $F\left(T_{r}\right)=\mathrm{EP}(F)$;

(4) $\mathrm{EP}(F)$ is closed and convex.

In the following, we introduce the property of $W$-mapping generated by a family of infinitely nonexpansive mappings.

Lemma 2.4 [14] Let $\left\{S_{i}: C \rightarrow C\right\}$ be a family of infinitely nonexpansive mappings with a nonempty common fixed point set, and let $\left\{\gamma_{i}\right\}$ be a real sequence such that $0<\gamma_{i} \leq l<1$, where $l$ is some real number, $\forall i>1$. Then

(1) $W_{n}$ is nonexpansive and $F\left(W_{n}\right)=\bigcap_{i=1}^{n} F\left(S_{i}\right)$ for each $n \geq 1$;

(2) for each $x \in C$ and for each positive integer $k$, the limit $\lim _{n \rightarrow \infty} U_{n, k}$ exists;

(3) the mapping $W: C \rightarrow C$ defined by

$$
W x:=\lim _{n \rightarrow \infty} W_{n} x=\lim _{n \rightarrow \infty} U_{n, 1} x, \quad x \in C,
$$

is a nonexpansive mapping satisfying $F(W)=\bigcap_{i=1}^{\infty} F\left(S_{i}\right)$ and it is called the $W$-mapping generated by $S_{1}, S_{2}, \ldots$ and $\gamma_{1}, \gamma_{2}, \ldots$. 
Lemma 2.5 [7] Let $\left\{S_{i}: C \rightarrow C\right\}$ be a family of infinitely nonexpansive mappings with a nonempty common fixed point set, and let $\left\{\gamma_{i}\right\}$ be a real sequence such that $0<\gamma_{i} \leq l<1$, $\forall i>1$. If $K$ is any bounded subset of $C$, then

$$
\limsup _{n \rightarrow \infty, x \in K}\left\|W x-W_{n} x\right\|=0
$$

The next lemma which we introduce is about the resolvent of the maximal monotone operator.

Lemma 2.6 (see [20-22]) Let $H$ be a real Hilbert space, and let $B$ be a maximal monotone operator on $H$. For $r>0$ and $x \in H$, define the resolvent $J_{r} x$. Then the following holds:

$$
\frac{s-t}{s}\left\langle J_{s} x-J_{t} x, J_{s} x-x\right\rangle \geq\left\|J_{s} x-J_{t} x\right\|^{2}
$$

for all $s, t>0$ and $x \in H$. In particular,

$$
\left\|J_{s} x-J_{t} x\right\| \leq(|s-t| / s)\left\|x-J_{s} x\right\|
$$

for all $s, t>0$ and $x \in H$.

The following lemma will be used in the proof of the main results.

Lemma 2.7 [23] Let $\left\{x_{n}\right\}$ and $\left\{y_{n}\right\}$ be bounded sequences in $H$, and let $\left\{\beta_{n}\right\}$ be a sequence in $(0,1)$ with $0<\liminf _{n \rightarrow \infty} \beta_{n} \leq \limsup _{n \rightarrow \infty} \beta_{n}<1$. Suppose that $x_{n+1}=\left(1-\beta_{n}\right) y_{n}+\beta_{n} x_{n}$ for all $n \geq 0$ and

$$
\limsup _{n \rightarrow \infty}\left(\left\|y_{n+1}-y_{n}\right\|-\left\|x_{n+1}-x_{n}\right\|\right) \leq 0
$$

Then $\lim _{n \rightarrow \infty}\left\|y_{n}-x_{n}\right\|=0$.

Besides, the following two lemmas are extremely important in the proof of theorems. One is called a demiclosed principle for nonexpansive mappings, the other is called an important lemma.

Lemma 2.8 (Demiclosed principle [24]) Let $T: C \rightarrow C$ be a nonexpansive mapping with $F(T) \neq \emptyset$. If $\left\{x_{n}\right\}_{n=1}^{\infty}$ is a sequence in $C$ weekly converging to $x$ and if $\left\{(I-T) x_{n}\right\}_{n=1}^{\infty}$ converges strongly to $y$, then $(I-T) x=y$. In particular, if $y=0$, then $x \in F(T)$.

Lemma 2.9 [25] Assume that $\left\{a_{n}\right\}_{n=0}^{\infty}$ is a sequence of nonnegative real numbers such that

$$
a_{n+1} \leq\left(1-\gamma_{n}\right) a_{n}+\gamma_{n} \delta_{n}+\beta_{n}, \quad n \geq 0,
$$

where $\left\{\gamma_{n}\right\}_{n=0}^{\infty}$ and $\left\{\beta_{n}\right\}_{n=0}^{\infty}$ are sequences in $(0,1)$ and $\left\{\delta_{n}\right\}_{n=0}^{\infty}$ is a sequence in $\mathbb{R}$ such that

(i) $\sum_{n=0}^{\infty} \gamma_{n}=\infty$;

(ii) either $\limsup _{n \rightarrow \infty} \delta_{n} \leq 0$ or $\sum_{n=0}^{\infty} \gamma_{n}\left|\delta_{n}\right|<\infty$;

(iii) $\sum_{n=0}^{\infty} \beta_{n}<\infty$.

Then $\lim _{n \rightarrow \infty} a_{n}=0$. 


\section{Main results}

Theorem 3.1 Let $H$ be a real Hilbert space, and let $C$ be a nonempty closed convex subset of $H$. Let $F: C \times C \rightarrow \mathbb{R}$ be a bifunction which satisfies (A1)-(A4), and let $f: C \rightarrow C$ be a $k$-contraction with the constant $k \in(0,1)$. Let $A: C \rightarrow H$ be an $\alpha$-inverse-strongly monotone $(\alpha$-ism) mapping with $\alpha>0$, let $B: C \rightarrow H$ be a $\beta$-inverse-strongly monotone $(\beta$-ism) mapping with $\beta>0$, and let $T: C \rightarrow H$ be a $\tau$-inverse-strongly monotone $(\tau$-ism) mapping with $\tau>0$. Let $M$ be a maximal monotone operator on $H$ such that the domain of $M$ is included in $C$, and let $J_{s}=(I+s M)^{-1}$ be the resolvent of $M$ for $s>0$. Let $N$ be a maximal monotone operator on $H$ such that the domain of $N$ is included in $C$, and let $J_{r}=(I+r N)^{-1}$ be the resolvent of $N$ for $r>0$. Let $\left\{S_{i}: C \rightarrow C\right\}$ be a family of infinitely nonexpansive mappings. Assume that $\Omega=\operatorname{GEP}(F, T) \cap \bigcap_{i=1}^{\infty} F\left(S_{i}\right) \cap(A+N)^{-1} 0 \cap(B+M)^{-1} 0 \neq \emptyset$. Let $\left\{\alpha_{n}\right\},\left\{\beta_{n}\right\}$ and $\left\{\gamma_{n}\right\}$ be sequences in $(0,1)$ such that $\alpha_{n}+\beta_{n}+\gamma_{n}=1$. Let $\left\{r_{n}\right\},\left\{\lambda_{n}\right\}$ and $\left\{s_{n}\right\}$ be positive number sequences. Let $x_{1} \in C$ and let $\left\{x_{n}\right\}$ be a sequence generated by

$$
\left\{\begin{array}{l}
y_{n}=J_{r_{n}}\left(u_{n}-r_{n} A u_{n}\right), \\
x_{n+1}=\alpha_{n} f\left(y_{n}\right)+\beta_{n} W_{n} J_{s_{n}}\left(y_{n}-s_{n} B y_{n}\right)+\gamma_{n} x_{n}, \quad \forall n \geq 1,
\end{array}\right.
$$

where $\left\{u_{n}\right\}$ is such that

$$
F\left(u_{n}, y\right)+\left\langle T x_{n}, y-u_{n}\right\rangle+\frac{1}{\lambda_{n}}\left\langle y-u_{n}, u_{n}-x_{n}\right\rangle \geq 0, \quad \forall y \in C,
$$

and $\left\{W_{n}\right\}$ is the sequence generated in (2.1). Assume that the following conditions hold:

(i) $0<a \leq r_{n} \leq b<2 \alpha$ and $\lim _{n \rightarrow \infty}\left|r_{n+1}-r_{n}\right|=0$;

(ii) $0<c \leq \lambda_{n} \leq d<2 \tau$ and $\lim _{n \rightarrow \infty}\left|\lambda_{n+1}-\lambda_{n}\right|=0$;

(iii) $0<e \leq s_{n} \leq g<2 \beta$ and $\lim _{n \rightarrow \infty}\left|s_{n+1}-s_{n}\right|=0$;

(iv) $\lim _{n \rightarrow \infty} \alpha_{n}=0, \sum_{n=1}^{\infty} \alpha_{n}=\infty$;

(v) $0<\liminf _{n \rightarrow \infty} \gamma_{n} \leq \limsup _{n \rightarrow \infty} \gamma_{n}<1$,

where $a, b, c, d, e$, and $g$ are real constants. Then $\left\{x_{n}\right\}$ converges strongly to a point $q \in \Omega$, which solves uniquely the following variational inequality:

$$
\langle q-f(q), q-x\rangle \leq 0, \quad \forall x \in \Omega
$$

Equivalently, $q=P_{\Omega_{2}} f(q)$.

Proof We divide the proof into several steps.

Step 1 . We prove that the sequence $\left\{x_{n}\right\}$ is bounded.

Since $A$ is an $\alpha$-ism mapping, we see from restriction (i) that $\forall x, y \in C$,

$$
\begin{aligned}
\left\|\left(I-r_{n} A\right) x-\left(I-r_{n} A\right) y\right\|^{2} & =\|x-y\|^{2}-2 r_{n}\langle x-y, A x-A y\rangle+r_{n}^{2}\|A x-A y\|^{2} \\
& \leq\|x-y\|^{2}-2 r_{n} \alpha\|A x-A y\|^{2}+r_{n}^{2}\|A x-A y\|^{2} \\
& =\|x-y\|^{2}+r_{n}\left(r_{n}-2 \alpha\right)\|A x-A y\|^{2} \\
& \leq\|x-y\|^{2} .
\end{aligned}
$$

This implies that $I-r_{n} A$ is nonexpansive. 
In the same way, we find that $I-s_{n} B$ and $I-\lambda_{n} T$ are nonexpansive. Note that $u_{n}=$ $T_{\lambda_{n}}\left(I-\lambda_{n} T\right) x_{n}$. Let $p \in \Omega$, it follows that

$$
\left\|u_{n}-p\right\| \leq\left\|\left(I-\lambda_{n} T\right) x_{n}-\left(I-\lambda_{n} T\right) p\right\| \leq\left\|x_{n}-p\right\|
$$

Putting $z_{n}=J_{s_{n}}\left(y_{n}-s_{n} B y_{n}\right)$, we see that

$$
\left\|z_{n}-p\right\| \leq\left\|y_{n}-p\right\| \leq\left\|u_{n}-p\right\| \leq\left\|x_{n}-p\right\|
$$

From (3.3), we find that

$$
\begin{aligned}
\left\|x_{n+1}-p\right\| & \leq \alpha_{n}\left\|f\left(y_{n}\right)-p\right\|+\beta_{n}\left\|W_{n} z_{n}-p\right\|+\gamma_{n}\left\|x_{n}-p\right\| \\
& \leq \alpha_{n} k\left\|y_{n}-p\right\|+\alpha_{n}\|f(p)-p\|+\beta_{n}\left\|z_{n}-p\right\|+\gamma_{n}\left\|x_{n}-p\right\| \\
& \leq\left(1-\alpha_{n}(1-k)\right)\left\|x_{n}-p\right\|+\alpha_{n}(1-k) \frac{\|f(p)-p\|}{1-k} .
\end{aligned}
$$

By induction, we derive that

$$
\left\|x_{n}-p\right\| \leq \max \left\{\left\|x_{1}-p\right\|, \frac{\|f(p)-p\|}{1-k}\right\}, \quad \forall n \geq 1
$$

Therefore, it turns out that $\left\{x_{n}\right\}$ is bounded, and so are $\left\{y_{n}\right\},\left\{z_{n}\right\}$ and $\left\{u_{n}\right\}$.

Step 2. We show that $\lim _{n \rightarrow \infty}\left\|x_{n+1}-x_{n}\right\|=0$.

Without loss of generality, we can assume that there exists a bounded set $K \subset C$ such that $x_{n}, y_{n}, z_{n}, u_{n} \in K$. Since $u_{n}=T_{\lambda_{n}}\left(I-\lambda_{n} T\right) x_{n}$, we find that

$$
F\left(u_{n+1}, y\right)+\frac{1}{\lambda_{n+1}}\left\langle y-u_{n+1}, u_{n+1}-\left(I-\lambda_{n+1} T\right) x_{n+1}\right\rangle \geq 0, \quad \forall y \in C
$$

and

$$
F\left(u_{n}, y\right)+\frac{1}{\lambda_{n}}\left\langle y-u_{n}, u_{n}-\left(I-\lambda_{n} T\right) x_{n}\right\rangle \geq 0, \quad \forall y \in C .
$$

Let $y=u_{n}$ in (3.4) and $y=u_{n+1}$ in (3.5). Then we add up (3.4) and (3.5) to derive that

$$
\left\langle u_{n+1}-u_{n}, u_{n}-u_{n+1}+u_{n+1}-\left(I-\lambda_{n} T\right) x_{n}-\frac{\lambda_{n}}{\lambda_{n+1}}\left(u_{n+1}-\left(I-\lambda_{n+1} T\right) x_{n+1}\right)\right\rangle \geq 0 .
$$

This implies that

$$
\begin{aligned}
\left\|u_{n+1}-u_{n}\right\|^{2} \leq & \left\langle u_{n+1}-u_{n},\left(I-\lambda_{n+1} T\right) x_{n+1}-\left(I-\lambda_{n} T\right) x_{n}\right. \\
& \left.+\left(1-\frac{\lambda_{n}}{\lambda_{n+1}}\right) \cdot\left(u_{n+1}-\left(I-\lambda_{n+1} T\right) x_{n+1}\right)\right\rangle \\
\leq & \left\|u_{n+1}-u_{n}\right\| \cdot\left(\left\|\left(I-\lambda_{n+1} T\right) x_{n+1}-\left(I-\lambda_{n} T\right) x_{n}\right\|\right. \\
& \left.+\left|1-\frac{\lambda_{n}}{\lambda_{n+1}}\right| \cdot\left\|u_{n+1}-\left(I-\lambda_{n+1} T\right) x_{n+1}\right\|\right) .
\end{aligned}
$$


From $I-\lambda_{n} T$ is nonexpansive and condition (ii), we obtain that

$$
\begin{aligned}
\left\|u_{n+1}-u_{n}\right\| \leq & \left\|\left(I-\lambda_{n+1} T\right) x_{n+1}-\left(I-\lambda_{n} T\right) x_{n}\right\| \\
& +\frac{\left|\lambda_{n+1}-\lambda_{n}\right|}{\lambda_{n+1}}\left\|u_{n+1}-\left(I-\lambda_{n+1} T\right) x_{n+1}\right\| \\
\leq & \left\|\left(I-\lambda_{n+1} T\right) x_{n+1}-\left(I-\lambda_{n+1} T\right) x_{n}\right\|+\left|\lambda_{n+1}-\lambda_{n}\right| \cdot\left\|T x_{n}\right\| \\
& +\frac{\left|\lambda_{n+1}-\lambda_{n}\right|}{c}\left\|u_{n+1}-\left(I-\lambda_{n+1} T\right) x_{n+1}\right\| \\
\leq & \left\|x_{n+1}-x_{n}\right\|+\left|\lambda_{n+1}-\lambda_{n}\right| L_{1},
\end{aligned}
$$

where $L_{1}$ is an appropriate constant such that

$$
L_{1}=\sup _{n \geq 1}\left\{\left\|T x_{n}\right\|+\frac{1}{c}\left\|u_{n+1}-\left(I-\lambda_{n+1} T\right) x_{n+1}\right\|\right\} .
$$

Since both $J_{r_{n}}$ and $I-r_{n} A$ are nonexpansive, it follows from Lemma 2.6, condition (i) and (3.6) that

$$
\begin{aligned}
\left\|y_{n+1}-y_{n}\right\|= & \left\|J_{r_{n+1}}\left(I-r_{n+1} A\right) u_{n+1}-J_{r_{n}}\left(I-r_{n} A\right) u_{n}\right\| \\
\leq & \left\|J_{r_{n+1}}\left(I-r_{n+1} A\right) u_{n+1}-J_{r_{n+1}}\left(I-r_{n+1} A\right) u_{n}\right\| \\
& +\left\|J_{r_{n+1}}\left(I-r_{n+1} A\right) u_{n}-J_{r_{n+1}}\left(I-r_{n} A\right) u_{n}\right\| \\
& +\left\|J_{r_{n+1}}\left(I-r_{n} A\right) u_{n}-J_{r_{n}}\left(I-r_{n} A\right) u_{n}\right\| \\
\leq & \left\|u_{n+1}-u_{n}\right\|+\left\|\left(I-r_{n+1} A\right) u_{n}-\left(I-r_{n} A\right) u_{n}\right\| \\
& +\frac{\left|r_{n+1}-r_{n}\right|}{r_{n+1}}\left\|J_{r_{n+1}}\left(I-r_{n} A\right) u_{n}-\left(I-r_{n} A\right) u_{n}\right\| \\
\leq & \left\|u_{n+1}-u_{n}\right\|+\left|r_{n+1}-r_{n}\right| \cdot\left\|A u_{n}\right\| \\
& +\frac{\left|r_{n+1}-r_{n}\right|}{a}\left\|J_{r_{n+1}}\left(I-r_{n} A\right) u_{n}-\left(I-r_{n} A\right) u_{n}\right\| \\
\leq & \left\|x_{n+1}-x_{n}\right\|+\left|\lambda_{n+1}-\lambda_{n}\right| L_{1}+\left|r_{n+1}-r_{n}\right| L_{2},
\end{aligned}
$$

where $L_{2}$ is an appropriate constant such that

$$
L_{2}=\sup _{n \geq 1}\left\{\left\|A u_{n}\right\|+\frac{1}{a}\left\|J_{r_{n+1}}\left(I-r_{n} A\right) u_{n}-\left(I-r_{n} A\right) u_{n}\right\|\right\} .
$$

Thus, from both $J_{s_{n}}$ and $I-s_{n} B$ are nonexpansive, we have from Lemma 2.6, condition (iii) and (3.7) that

$$
\begin{aligned}
\left\|z_{n+1}-z_{n}\right\|= & \left\|J_{s_{n+1}}\left(I-s_{n+1} B\right) y_{n+1}-J_{s_{n}}\left(I-s_{n} B\right) y_{n}\right\| \\
\leq & \left\|J_{s_{n+1}}\left(I-s_{n+1} B\right) y_{n+1}-J_{s_{n+1}}\left(I-s_{n+1} B\right) y_{n}\right\| \\
& \quad+\left\|J_{s_{n+1}}\left(I-s_{n+1} B\right) y_{n}-J_{s_{n+1}}\left(I-s_{n} B\right) y_{n}\right\| \\
& \quad+\left\|J_{s_{n+1}}\left(I-s_{n} B\right) y_{n}-J_{s_{n}}\left(I-s_{n} B\right) y_{n}\right\| \\
\leq & \left\|y_{n+1}-y_{n}\right\|+\left\|\left(I-s_{n+1} B\right) y_{n}-\left(I-s_{n} B\right) y_{n}\right\|
\end{aligned}
$$




$$
\begin{aligned}
& +\frac{\left|s_{n+1}-s_{n}\right|}{s_{n+1}}\left\|J_{s_{n+1}}\left(I-s_{n} B\right) y_{n}-\left(I-s_{n} B\right) y_{n}\right\| \\
\leq & \left\|y_{n+1}-y_{n}\right\|+\left|s_{n+1}-s_{n}\right| \cdot\left\|B y_{n}\right\| \\
& +\frac{\left|s_{n+1}-s_{n}\right|}{e}\left\|J_{s_{n+1}}\left(I-s_{n} B\right) y_{n}-\left(I-s_{n} B\right) y_{n}\right\| \\
\leq & \left\|x_{n+1}-x_{n}\right\| \\
& +L_{3}\left(\left|\lambda_{n+1}-\lambda_{n}\right|+\left|r_{n+1}-r_{n}\right|+\left|s_{n+1}-s_{n}\right|\right),
\end{aligned}
$$

where $L_{3}=\max \left\{L_{1}, L_{2}, \sup _{n \geq 1}\left\{\left\|B y_{n}\right\|+\frac{1}{e}\left\|J_{s_{n+1}}\left(I-s_{n} B\right) y_{n}-\left(I-s_{n} B\right) y_{n}\right\|\right\}\right\}$.

It yields from (3.8) that

$$
\begin{aligned}
\left\|W_{n+1} z_{n+1}-W_{n} z_{n}\right\| \leq & \left\|W_{n+1} z_{n+1}-W z_{n+1}\right\|+\left\|W z_{n+1}-W z_{n}\right\| \\
& +\left\|W z_{n}-W_{n} z_{n}\right\| \\
\leq & \sup _{x \in K}\left\{\left\|W_{n+1} x-W x\right\|+\left\|W x-W_{n} x\right\|\right\}+\left\|x_{n+1}-x_{n}\right\| \\
& +L_{3}\left(\left|\lambda_{n+1}-\lambda_{n}\right|+\left|r_{n+1}-r_{n}\right|+\left|s_{n+1}-s_{n}\right|\right),
\end{aligned}
$$

where $K$ is the bounded subset of $C$ defined above. Let $x_{n+1}=\left(1-\gamma_{n}\right) t_{n}+\gamma_{n} x_{n}$. It follows that

$$
\begin{aligned}
t_{n+1}-t_{n}= & \frac{\alpha_{n+1} f\left(y_{n+1}\right)+\beta_{n+1} W_{n+1} z_{n+1}}{1-\gamma_{n+1}}-\frac{\alpha_{n} f\left(y_{n}\right)+\beta_{n} W_{n} z_{n}}{1-\gamma_{n}} \\
= & \frac{\alpha_{n+1}}{1-\gamma_{n+1}} f\left(y_{n+1}\right)+\frac{1-\alpha_{n+1}-\gamma_{n+1}}{1-\gamma_{n+1}} W_{n+1} z_{n+1} \\
& -\left(\frac{\alpha_{n}}{1-\gamma_{n}} f\left(y_{n}\right)+\frac{1-\alpha_{n}-\gamma_{n}}{1-\gamma_{n}} W_{n} z_{n}\right) \\
= & \frac{\alpha_{n+1}}{1-\gamma_{n+1}}\left(f\left(y_{n+1}\right)-W_{n+1} z_{n+1}\right)-\frac{\alpha_{n}}{1-\gamma_{n}}\left(f\left(y_{n}\right)-W_{n} z_{n}\right) \\
& +W_{n+1} z_{n+1}-W_{n} z_{n} .
\end{aligned}
$$

From (3.9), we derive that

$$
\begin{aligned}
\left\|t_{n+1}-t_{n}\right\| \leq & \frac{\alpha_{n+1}}{1-\gamma_{n+1}}\left\|f\left(y_{n+1}\right)-W_{n+1} z_{n+1}\right\|+\frac{\alpha_{n}}{1-\gamma_{n}}\left\|f\left(y_{n}\right)-W_{n} z_{n}\right\| \\
& +\left\|W_{n+1} z_{n+1}-W_{n} z_{n}\right\| \\
\leq & \frac{\alpha_{n+1}}{1-\gamma_{n+1}}\left\|f\left(y_{n+1}\right)-W_{n+1} z_{n+1}\right\|+\frac{\alpha_{n}}{1-\gamma_{n}}\left\|f\left(y_{n}\right)-W_{n} z_{n}\right\| \\
& +\sup _{x \in K}\left\{\left\|W_{n+1} x-W x\right\|+\left\|W x-W_{n} x\right\|\right\}+\left\|x_{n+1}-x_{n}\right\| \\
& +L_{3}\left(\left|\lambda_{n+1}-\lambda_{n}\right|+\left|r_{n+1}-r_{n}\right|+\left|s_{n+1}-s_{n}\right|\right),
\end{aligned}
$$

which implies that

$$
\begin{aligned}
\left\|t_{n+1}-t_{n}\right\|-\left\|x_{n+1}-x_{n}\right\| \leq & \frac{\alpha_{n+1}}{1-\gamma_{n+1}}\left\|f\left(y_{n+1}\right)-W_{n+1} z_{n+1}\right\| \\
& +\frac{\alpha_{n}}{1-\gamma_{n}}\left\|f\left(y_{n}\right)-W_{n} z_{n}\right\|
\end{aligned}
$$




$$
\begin{aligned}
& +\sup _{x \in K}\left\{\left\|W_{n+1} x-W x\right\|+\left\|W x-W_{n} x\right\|\right\} \\
& +L_{3}\left(\left|\lambda_{n+1}-\lambda_{n}\right|+\left|r_{n+1}-r_{n}\right|+\left|s_{n+1}-s_{n}\right|\right) .
\end{aligned}
$$

It follows from conditions (i)-(v) that

$$
\lim _{n \rightarrow \infty} \sup \left(\left\|t_{n+1}-t_{n}\right\|-\left\|x_{n+1}-x_{n}\right\|\right) \leq 0 .
$$

From Lemma 2.7, we derive that $\lim _{n \rightarrow \infty}\left\|t_{n}-x_{n}\right\|=0$.

Since $t_{n}-x_{n}=\frac{1}{1-\gamma_{n}}\left(x_{n+1}-x_{n}\right)$, it follows that

$$
\lim _{n \rightarrow \infty}\left\|x_{n+1}-x_{n}\right\|=0 .
$$

Step 3. We prove that $\lim _{n \rightarrow \infty}\left\|x_{n}-u_{n}\right\|=0$.

Since $T$ is $\tau$-ism, we find from (3.3) that

$$
\begin{aligned}
\left\|x_{n+1}-p\right\|^{2} & \leq \alpha_{n}\left\|f\left(y_{n}\right)-p\right\|^{2}+\beta_{n}\left\|u_{n}-p\right\|^{2}+\gamma_{n}\left\|x_{n}-p\right\|^{2} \\
& \leq \alpha_{n}\left\|f\left(y_{n}\right)-p\right\|^{2}+\beta_{n}\left\|x_{n}-p-\lambda_{n}\left(T x_{n}-T p\right)\right\|^{2}+\gamma_{n}\left\|x_{n}-p\right\|^{2} \\
& \leq \alpha_{n}\left\|f\left(y_{n}\right)-p\right\|^{2}+\left\|x_{n}-p\right\|^{2}-\lambda_{n} \beta_{n}\left(2 \tau-\lambda_{n}\right)\left\|T x_{n}-T p\right\|^{2},
\end{aligned}
$$

it turns out that

$$
\begin{aligned}
& \lambda_{n} \beta_{n}\left(2 \tau-\lambda_{n}\right)\left\|T x_{n}-T p\right\|^{2} \\
& \quad \leq \alpha_{n}\left\|f\left(y_{n}\right)-p\right\|^{2}+\left(\left\|x_{n}-p\right\|+\left\|x_{n+1}-p\right\|\right)\left\|x_{n+1}-x_{n}\right\| .
\end{aligned}
$$

By virtue of conditions (ii), (iv), (v), we derive from (3.10) that

$$
\lim _{n \rightarrow \infty}\left\|T x_{n}-T p\right\|=0
$$

Since $T_{\lambda_{n}}$ is firmly nonexpansive, we find from Lemma 2.3 that

$$
\begin{aligned}
\left\|u_{n}-p\right\|^{2}= & \left\|T_{\lambda_{n}}\left(I-\lambda_{n} T\right) x_{n}-T_{\lambda_{n}}\left(I-\lambda_{n} T\right) p\right\|^{2} \\
\leq & \left\langle\left(I-\lambda_{n} T\right) x_{n}-\left(I-\lambda_{n} T\right) p, u_{n}-p\right\rangle \\
\leq & \frac{1}{2}\left(\left\|x_{n}-p\right\|^{2}+\left\|u_{n}-p\right\|^{2}-\left\|x_{n}-u_{n}\right\|^{2}\right. \\
& \left.+2 \lambda_{n}\left\|T x_{n}-T p\right\| \cdot\left\|x_{n}-u_{n}\right\|\right),
\end{aligned}
$$

it implies that

$$
\left\|u_{n}-p\right\|^{2} \leq\left\|x_{n}-p\right\|^{2}-\left\|x_{n}-u_{n}\right\|^{2}+2 \lambda_{n}\left\|T x_{n}-T p\right\| \cdot\left\|x_{n}-u_{n}\right\| .
$$

Therefore, we derive that

$$
\begin{aligned}
\left\|x_{n+1}-p\right\|^{2} \leq & \alpha_{n}\left\|f\left(y_{n}\right)-p\right\|^{2}+\beta_{n}\left\|u_{n}-p\right\|^{2}+\gamma_{n}\left\|x_{n}-p\right\|^{2} \\
\leq & \alpha_{n}\left\|f\left(y_{n}\right)-p\right\|^{2}-\beta_{n}\left\|x_{n}-u_{n}\right\|^{2} \\
& +2 \lambda_{n} \beta_{n}\left\|T x_{n}-T p\right\| \cdot\left\|x_{n}-u_{n}\right\|+\left\|x_{n}-p\right\|^{2},
\end{aligned}
$$


which yields that

$$
\begin{aligned}
\beta_{n}\left\|x_{n}-u_{n}\right\|^{2} \leq & \alpha_{n}\left\|f\left(y_{n}\right)-p\right\|^{2}+2 \lambda_{n} \beta_{n}\left\|T x_{n}-T p\right\| \cdot\left\|x_{n}-u_{n}\right\| \\
& +\left(\left\|x_{n}-p\right\|+\left\|x_{n+1}-p\right\|\right)\left\|x_{n+1}-x_{n}\right\| .
\end{aligned}
$$

By use of conditions (ii), (iv), (v), we obtain from (3.10) and (3.11) that

$$
\lim _{n \rightarrow \infty}\left\|x_{n}-u_{n}\right\|=0 .
$$

Step 4. We prove that $\lim _{n \rightarrow \infty}\left\|u_{n}-y_{n}\right\|=0$.

Since $J_{r_{n}}$ is nonexpansive, $A$ is $\alpha$-ism, we find from (3.3) that

$$
\begin{aligned}
\left\|y_{n}-p\right\|^{2} & =\left\|J_{r_{n}}\left(I-r_{n} A\right) u_{n}-J_{r_{n}}\left(I-r_{n} A\right) p\right\|^{2} \\
& \leq\left\|\left(I-r_{n} A\right) u_{n}-\left(I-r_{n} A\right) p\right\|^{2} \\
& \leq\left\|u_{n}-p\right\|^{2}-2 r_{n} \alpha\left\|A u_{n}-A p\right\|^{2}+r_{n}^{2}\left\|A u_{n}-A p\right\|^{2} \\
& \leq\left\|x_{n}-p\right\|^{2}+r_{n}\left(r_{n}-2 \alpha\right)\left\|A u_{n}-A p\right\|^{2} .
\end{aligned}
$$

Therefore, we derive that

$$
\begin{aligned}
\left\|x_{n+1}-p\right\|^{2} & \leq \alpha_{n}\left\|f\left(y_{n}\right)-p\right\|^{2}+\beta_{n}\left\|W_{n} z_{n}-p\right\|^{2}+\gamma_{n}\left\|x_{n}-p\right\|^{2} \\
& \leq \alpha_{n}\left\|f\left(y_{n}\right)-p\right\|^{2}+\beta_{n}\left\|y_{n}-p\right\|^{2}+\gamma_{n}\left\|x_{n}-p\right\|^{2} \\
& \leq \alpha_{n}\left\|f\left(y_{n}\right)-p\right\|^{2}+r_{n}\left(r_{n}-2 \alpha\right) \beta_{n}\left\|A u_{n}-A p\right\|^{2}+\left\|x_{n}-p\right\|^{2},
\end{aligned}
$$

it turns out that

$$
\begin{aligned}
& r_{n}\left(2 \alpha-r_{n}\right) \beta_{n}\left\|A u_{n}-A p\right\|^{2} \\
& \quad \leq \alpha_{n}\left\|f\left(y_{n}\right)-p\right\|^{2}+\left(\left\|x_{n}-p\right\|+\left\|x_{n+1}-p\right\|\right)\left\|x_{n+1}-x_{n}\right\| .
\end{aligned}
$$

In view of conditions (i), (iv), (v), we obtain from (3.10) that

$$
\lim _{n \rightarrow \infty}\left\|A u_{n}-A p\right\|=0 .
$$

Since $J_{r_{n}}$ is firmly nonexpansive, $I-r_{n} A$ is nonexpansive, we find from (3.3) that

$$
\begin{aligned}
\left\|y_{n}-p\right\|^{2}= & \left\|J_{r_{n}}\left(I-r_{n} A\right) u_{n}-J_{r_{n}}\left(I-r_{n} A\right) p\right\|^{2} \\
\leq & \left\langle\left(I-r_{n} A\right) u_{n}-\left(I-r_{n} A\right) p, y_{n}-p\right\rangle \\
= & \frac{1}{2}\left\{\left\|\left(I-r_{n} A\right) u_{n}-\left(I-r_{n} A\right) p\right\|^{2}+\left\|y_{n}-p\right\|^{2}\right. \\
& \left.-\left\|\left(I-r_{n} A\right) u_{n}-\left(I-r_{n} A\right) p-\left(y_{n}-p\right)\right\|^{2}\right\} \\
\leq & \frac{1}{2}\left\{\left\|u_{n}-p\right\|^{2}+\left\|y_{n}-p\right\|^{2}-\left\|u_{n}-y_{n}-r_{n}\left(A u_{n}-A p\right)\right\|^{2}\right\} \\
\leq & \frac{1}{2}\left\{\left\|x_{n}-p\right\|^{2}+\left\|y_{n}-p\right\|^{2}-\left\|u_{n}-y_{n}\right\|^{2}\right. \\
& \left.+2 r_{n}\left\|u_{n}-y_{n}\right\| \cdot\left\|A u_{n}-A p\right\|\right\},
\end{aligned}
$$


which implies that

$$
\left\|y_{n}-p\right\|^{2} \leq\left\|x_{n}-p\right\|^{2}-\left\|u_{n}-y_{n}\right\|^{2}+2 r_{n}\left\|u_{n}-y_{n}\right\| \cdot\left\|A u_{n}-A p\right\| .
$$

From (3.3), this further implies that

$$
\begin{aligned}
\left\|x_{n+1}-p\right\|^{2} \leq & \alpha_{n}\left\|f\left(y_{n}\right)-p\right\|^{2}+\beta_{n}\left\|W_{n} z_{n}-p\right\|^{2}+\gamma_{n}\left\|x_{n}-p\right\|^{2} \\
\leq & \alpha_{n}\left\|f\left(y_{n}\right)-p\right\|^{2}+\beta_{n}\left\|y_{n}-p\right\|^{2}+\gamma_{n}\left\|x_{n}-p\right\|^{2} \\
\leq & \alpha_{n}\left\|f\left(y_{n}\right)-p\right\|^{2}-\beta_{n}\left\|u_{n}-y_{n}\right\|^{2} \\
& +2 r_{n} \beta_{n}\left\|u_{n}-y_{n}\right\| \cdot\left\|A u_{n}-A p\right\|+\left\|x_{n}-p\right\|^{2},
\end{aligned}
$$

which yields that

$$
\begin{gathered}
\beta_{n}\left\|u_{n}-y_{n}\right\|^{2} \leq \alpha_{n}\left\|f\left(y_{n}\right)-p\right\|^{2}+2 r_{n} \beta_{n}\left\|u_{n}-y_{n}\right\| \cdot\left\|A u_{n}-A p\right\| \\
+\left(\left\|x_{n}-p\right\|+\left\|x_{n+1}-p\right\|\right)\left\|x_{n+1}-x_{n}\right\| .
\end{gathered}
$$

By use of conditions (i), (iv), (v), we derive from (3.10) and (3.13) that

$$
\lim _{n \rightarrow \infty}\left\|u_{n}-y_{n}\right\|=0
$$

Step 5. We prove that $\lim _{n \rightarrow \infty}\left\|y_{n}-z_{n}\right\|=0$.

Since $J_{s_{n}}$ is nonexpansive, $B$ is $\beta$-ism, we find from (3.3) that

$$
\begin{aligned}
\left\|z_{n}-p\right\|^{2} & =\left\|J_{s_{n}}\left(I-s_{n} B\right) y_{n}-J_{s_{n}}\left(I-s_{n} B\right) p\right\|^{2} \\
& \leq\left\|\left(I-s_{n} B\right) y_{n}-\left(I-s_{n} B\right) p\right\|^{2} \\
& \leq\left\|y_{n}-p\right\|^{2}-2 s_{n} \beta\left\|B y_{n}-B p\right\|^{2}+s_{n}^{2}\left\|B y_{n}-B p\right\|^{2} \\
& \leq\left\|x_{n}-p\right\|^{2}+s_{n}\left(s_{n}-2 \beta\right)\left\|B y_{n}-B p\right\|^{2} .
\end{aligned}
$$

Hence, we derive that

$$
\begin{aligned}
\left\|x_{n+1}-p\right\|^{2} & =\left\|\alpha_{n}\left(f\left(y_{n}\right)-p\right)+\beta_{n}\left(W_{n} z_{n}-p\right)+\gamma_{n}\left(x_{n}-p\right)\right\|^{2} \\
& \leq \alpha_{n}\left\|f\left(y_{n}\right)-p\right\|^{2}+\beta_{n}\left\|z_{n}-p\right\|^{2}+\gamma_{n}\left\|x_{n}-p\right\|^{2} \\
& \leq \alpha_{n}\left\|f\left(y_{n}\right)-p\right\|^{2}+s_{n}\left(s_{n}-2 \beta\right) \beta_{n}\left\|B y_{n}-B p\right\|^{2}+\left\|x_{n}-p\right\|^{2},
\end{aligned}
$$

which implies that

$$
\begin{aligned}
& s_{n}\left(2 \beta-s_{n}\right) \beta_{n}\left\|B y_{n}-B p\right\|^{2} \\
& \quad \leq \alpha_{n}\left\|f\left(y_{n}\right)-p\right\|^{2}+\left(\left\|x_{n}-p\right\|+\left\|x_{n+1}-p\right\|\right)\left\|x_{n+1}-x_{n}\right\| .
\end{aligned}
$$

By virtue of conditions (iii), (iv), (v), we derive from (3.10) that

$$
\lim _{n \rightarrow \infty}\left\|B y_{n}-B p\right\|=0
$$


Since $J_{s_{n}}$ is firmly nonexpansive, $I-s_{n} B$ is nonexpansive, we find from (3.3) that

$$
\begin{aligned}
\left\|z_{n}-p\right\|^{2}= & \left\|J_{s_{n}}\left(I-s_{n} B\right) y_{n}-J_{s_{n}}\left(I-s_{n} B\right) p\right\|^{2} \\
\leq & \left\langle\left(I-s_{n} B\right) y_{n}-\left(I-s_{n} B\right) p, z_{n}-p\right\rangle \\
= & \frac{1}{2}\left\{\left\|\left(I-s_{n} B\right) y_{n}-\left(I-s_{n} B\right) p\right\|^{2}+\left\|z_{n}-p\right\|^{2}\right. \\
& \left.-\left\|\left(I-s_{n} B\right) y_{n}-\left(I-s_{n} B\right) p-\left(z_{n}-p\right)\right\|^{2}\right\} \\
\leq & \frac{1}{2}\left\{\left\|y_{n}-p\right\|^{2}+\left\|z_{n}-p\right\|^{2}-\left\|y_{n}-z_{n}-s_{n}\left(B y_{n}-B p\right)\right\|^{2}\right\} \\
\leq & \frac{1}{2}\left\{\left\|x_{n}-p\right\|^{2}+\left\|z_{n}-p\right\|^{2}-\left\|y_{n}-z_{n}\right\|^{2}\right. \\
& \left.+2 s_{n}\left\|y_{n}-z_{n}\right\| \cdot\left\|B y_{n}-B p\right\|\right\} .
\end{aligned}
$$

It turns out that

$$
\left\|z_{n}-p\right\|^{2} \leq\left\|x_{n}-p\right\|^{2}-\left\|y_{n}-z_{n}\right\|^{2}+2 s_{n}\left\|y_{n}-z_{n}\right\| \cdot\left\|B y_{n}-B p\right\| .
$$

Hence, we obtain that

$$
\begin{aligned}
\left\|x_{n+1}-p\right\|^{2} \leq & \alpha_{n}\left\|f\left(y_{n}\right)-p\right\|^{2}+\beta_{n}\left\|W_{n} z_{n}-p\right\|^{2}+\gamma_{n}\left\|x_{n}-p\right\|^{2} \\
\leq & \alpha_{n}\left\|f\left(y_{n}\right)-p\right\|^{2}+\beta_{n}\left\|z_{n}-p\right\|^{2}+\gamma_{n}\left\|x_{n}-p\right\|^{2} \\
\leq & \alpha_{n}\left\|f\left(y_{n}\right)-p\right\|^{2}-\beta_{n}\left\|y_{n}-z_{n}\right\|^{2} \\
& +2 s_{n} \beta_{n}\left\|y_{n}-z_{n}\right\| \cdot\left\|B y_{n}-B p\right\|+\left\|x_{n}-p\right\|^{2},
\end{aligned}
$$

it follows that

$$
\begin{aligned}
\beta_{n}\left\|y_{n}-z_{n}\right\|^{2} \leq & \alpha_{n}\left\|f\left(y_{n}\right)-p\right\|^{2}+2 s_{n} \beta_{n}\left\|y_{n}-z_{n}\right\| \cdot\left\|B y_{n}-B p\right\| \\
& +\left(\left\|x_{n}-p\right\|+\left\|x_{n+1}-p\right\|\right)\left\|x_{n+1}-x_{n}\right\| .
\end{aligned}
$$

By use of conditions (iii), (iv), (v), we obtain from (3.10) and (3.15) that

$$
\lim _{n \rightarrow \infty}\left\|y_{n}-z_{n}\right\|=0
$$

Step 6. We show that

$$
\lim _{n \rightarrow \infty} \sup \left\langle f(q)-q, x_{n}-q\right\rangle \leq 0, \quad \forall x_{n} \in \Omega
$$

where $q=P_{\Omega} f(q)$. It is equivalent to show that $q \in \Omega=\operatorname{GEP}(F, T) \cap \bigcap_{i=1}^{\infty} F\left(S_{i}\right) \cap(A+$ $N)^{-1} 0 \cap(B+M)^{-1} 0$.

First, we show that $q \in \operatorname{GEP}(F, T)$. From Lemma 2.3, we get $u_{n}=T_{\lambda_{n}}\left(I-\lambda_{n} T\right) x_{n}$, for any $y \in C$, we find from (A2) that

$$
\left\langle T x_{n_{i}}, y-u_{n_{i}}\right\rangle+\left\langle y-u_{n_{i}}, \frac{u_{n_{i}}-x_{n_{i}}}{\lambda_{n_{i}}}\right\rangle \geq F\left(y, u_{n_{i}}\right), \quad \forall y \in C .
$$


Putting $y_{h}=h y+(1-h) q$ for any $h \in(0,1]$ and $y \in C$, we see that $y_{h} \in C$. From (3.17), we derive that

$$
\begin{aligned}
\left\langle y_{h}-u_{n_{i}}, T y_{h}\right\rangle \geq & \left\langle y_{h}-u_{n_{i}}, T y_{h}\right\rangle-\left\langle T x_{n_{i}}, y_{h}-u_{n_{i}}\right\rangle \\
& -\left\langle y_{h}-u_{n_{i}}, \frac{u_{n_{i}}-x_{n_{i}}}{\lambda_{n_{i}}}\right\rangle+F\left(y_{h}, u_{n_{i}}\right) \\
= & \left\langle y_{h}-u_{n_{i}}, T y_{h}-T u_{n_{i}}\right\rangle+\left\langle y_{h}-u_{n_{i}}, T u_{n_{i}}-T x_{n_{i}}\right\rangle \\
& -\left\langle y_{h}-u_{n_{i}}, \frac{u_{n_{i}}-x_{n_{i}}}{\lambda_{n_{i}}}\right\rangle+F\left(y_{h}, u_{n_{i}}\right) .
\end{aligned}
$$

By virtue of the monotonicity of $T$ and condition (ii), we obtain from (A4) that

$$
\left\langle y_{h}-q, T y_{h}\right\rangle \geq F\left(y_{h}, q\right)
$$

From (A1) and (A4), we see that

$$
\begin{aligned}
0 & =F\left(y_{h}, y_{h}\right) \leq h F\left(y_{h}, y\right)+(1-h) F\left(y_{h}, q\right) \\
& \leq h F\left(y_{h}, y\right)+(1-h)\left\langle y_{h}-q, T y_{h}\right\rangle \\
& =h F\left(y_{h}, y\right)+(1-h) h\left\langle y-q, T y_{h}\right\rangle .
\end{aligned}
$$

It turns out from (A3) that $q \in \operatorname{GEP}(F, T)$.

Then we show that $q \in \bigcap_{i=1}^{\infty} F\left(S_{i}\right)$. Indeed, choose a subsequence $\left\{x_{n_{i}}\right\}$ of $\left\{x_{n}\right\}$ such that

$$
\lim _{n \rightarrow \infty} \sup \left\langle(f-I) q, x_{n}-q\right\rangle=\lim _{i \rightarrow \infty}\left\langle(f-I) q, x_{n_{i}}-q\right\rangle .
$$

Since $\left\{x_{n_{i}}\right\}$ is bounded, there exists a subsequence $\left\{x_{n_{i_{j}}}\right\}$ of $\left\{x_{n_{i}}\right\}$ such that $\left\{x_{n_{i_{j}}}\right\}$ converges weakly to $q$. Without loss of generality, we may assume that $x_{n_{i}} \rightarrow q$. In view of (3.12), (3.14) and (3.16), we know $x_{n_{i}} \rightarrow q$ is equivalent to $u_{n_{i}} \rightarrow q, y_{n_{i}} \rightarrow q$ and $z_{n_{i}} \rightarrow q$.

Since $x_{n+1}=\alpha_{n} f\left(y_{n}\right)+\beta_{n} W_{n} z_{n}+\gamma_{n} x_{n}$, it implies that

$$
\beta_{n}\left\|W_{n} z_{n}-x_{n}\right\| \leq\left\|x_{n+1}-x_{n}\right\|+\alpha_{n}\left\|f\left(y_{n}\right)-x_{n}\right\| .
$$

By virtue of conditions (iv), (v), we derive from (3.10) that

$$
\lim _{n \rightarrow \infty}\left\|W_{n} z_{n}-x_{n}\right\|=0 .
$$

Observing that

$$
\left\|W_{n} z_{n}-z_{n}\right\| \leq\left\|W_{n} z_{n}-x_{n}\right\|+\left\|x_{n}-u_{n}\right\|+\left\|u_{n}-y_{n}\right\|+\left\|y_{n}-z_{n}\right\|,
$$

and from (3.12), (3.14), (3.16) and (3.19), we derive that

$$
\lim _{n \rightarrow \infty}\left\|W_{n} z_{n}-z_{n}\right\|=0
$$

It is not hard to find that

$$
\left\|W z_{n}-z_{n}\right\| \leq\left\|W z_{n}-W_{n} z_{n}\right\|+\left\|W_{n} z_{n}-z_{n}\right\| \leq \sup _{x \in K}\left\|W x-W_{n} x\right\|+\left\|W_{n} z_{n}-z_{n}\right\| .
$$


From Lemma 2.5 and (3.20), we obtain that

$$
\lim _{n \rightarrow \infty}\left\|W z_{n}-z_{n}\right\|=0
$$

Since $z_{n_{i}} \rightarrow q, W: C \rightarrow C$ is a nonexpansive mapping, we get by Lemma 2.8 that $q \in F(W)$. Then by Lemma 2.4, we know that $q \in F(W)$ is equivalent to $q \in \bigcap_{i=1}^{\infty} F\left(S_{i}\right)$.

In the following, we show that $q \in(A+N)^{-1} 0$.

As in [26], we have that for any $r>0$,

$$
\begin{aligned}
q \in(A+N)^{-1} 0 & \Longleftrightarrow 0 \in A q+N q \\
& \Longleftrightarrow 0 \in r A q+r N q \\
& \Longleftrightarrow q-r A q \in q+r N q \\
& \Longleftrightarrow q=J_{r}(I-r A) q \\
& \Longleftrightarrow q \in \operatorname{Fix}\left(J_{r}(I-r A)\right) .
\end{aligned}
$$

In view of condition (i), we can take $r_{0} \in[a, b]$. From Lemma 2.6 and $J_{r_{0}}$ is nonexpansive, we derive that

$$
\begin{aligned}
\left\|J_{r_{0}}\left(I-r_{0} A\right) u_{n}-y_{n}\right\| \leq & \left\|J_{r_{0}}\left(I-r_{0} A\right) u_{n}-J_{r_{0}}\left(I-r_{n} A\right) u_{n}\right\| \\
& +\left\|J_{r_{0}}\left(I-r_{n} A\right) u_{n}-y_{n}\right\| \\
\leq & \left\|\left(I-r_{0} A\right) u_{n}-\left(I-r_{n} A\right) u_{n}\right\| \\
& +\left\|J_{r_{0}}\left(I-r_{n} A\right) u_{n}-J_{r_{n}}\left(I-r_{n} A\right) u_{n}\right\| \\
\leq & \left|r_{n}-r_{0}\right| \cdot\left\|A\left(u_{n}\right)\right\| \\
& +\frac{\left|r_{n}-r_{0}\right|}{r_{0}}\left\|J_{r_{0}}\left(I-r_{n} A\right) u_{n}-\left(I-r_{n} A\right) u_{n}\right\| \rightarrow 0 .
\end{aligned}
$$

Observing that

$$
\left\|J_{r_{0}}\left(I-r_{0} A\right) u_{n}-u_{n}\right\| \leq\left\|J_{r_{0}}\left(I-r_{0} A\right) u_{n}-y_{n}\right\|+\left\|y_{n}-u_{n}\right\|
$$

and from (3.14) and (3.22), we have that

$$
\left\|J_{r_{0}}\left(I-r_{0} A\right) u_{n}-u_{n}\right\| \rightarrow 0
$$

From the boundedness of $\left\{x_{n}\right\}$, we may assume that there exists a subsequence $\left\{x_{n_{i}}\right\}$ of $\left\{x_{n}\right\}$ such that $x_{n_{i}} \rightarrow q, q \in C$. By (3.12), we also have that $u_{n_{i}} \rightarrow q, q \in C$. On the other hand, from $r_{n} \rightarrow r_{0} \in[a, b]$, we have that $r_{n_{i}} \rightarrow r_{0} \in[a, b]$.

By use of (3.23), we have that

$$
\left\|J_{r_{0}}\left(I-r_{0} A\right) u_{n_{i}}-u_{n_{i}}\right\| \rightarrow 0 .
$$

Since $J_{r_{0}}\left(I-r_{0} A\right)$ is nonexpansive, we have from Lemma 2.8 that $q=J_{r_{0}}\left(I-r_{0} A\right) q$. In virtue of (3.21), this means that $q \in(A+N)^{-1} 0$. 
By use of the similar proof method, we can also derive that

$$
\left\|J_{s_{0}}\left(I-s_{0} B\right) y_{n}-z_{n}\right\| \rightarrow 0
$$

and we also have from (3.16) and (3.24) that

$$
\left\|J_{s_{0}}\left(I-s_{0} B\right) y_{n}-y_{n}\right\| \leq\left\|J_{s_{0}}\left(I-s_{0} B\right) y_{n}-z_{n}\right\|+\left\|y_{n}-z_{n}\right\| \rightarrow 0 .
$$

From the above proof processing, $x_{n_{i}} \rightarrow q$ and $\left\|x_{n}-y_{n}\right\| \rightarrow 0$, we have that $y_{n_{i}} \rightarrow q$, where $q \in C$. On the other hand, from $s_{n} \rightarrow s_{0} \in[e, g]$, we have that $s_{n_{i}} \rightarrow s_{0} \in[e, g]$.

In view of the above inequality, we have that

$$
\left\|J_{s_{0}}\left(I-s_{0} B\right) y_{n_{i}}-y_{n_{i}}\right\| \rightarrow 0
$$

Since $J_{s_{0}}\left(I-s_{0} B\right)$ is nonexpansive, we also have from Lemma 2.8 that $q=J_{s_{0}}\left(I-s_{0} B\right) q$. By (3.21), we obtain that $q \in(B+M)^{-1} 0$.

Step 7 . We finally prove that $x_{n} \rightarrow q$ in norm.

Indeed, we derive from (3.1) and (3.3) that

$$
\begin{aligned}
\left\|x_{n+1}-q\right\|^{2}= & \alpha_{n}\left\langle f\left(y_{n}\right)-q, x_{n+1}-q\right\rangle+\beta_{n}\left\langle W_{n} z_{n}-q, x_{n+1}-q\right\rangle \\
& +\gamma_{n}\left\langle x_{n}-q, x_{n+1}-q\right\rangle \\
\leq & \alpha_{n} k\left\|y_{n}-q\right\| \cdot\left\|x_{n+1}-q\right\|+\alpha_{n}\left\langle f(q)-q, x_{n+1}-q\right\rangle \\
& +\beta_{n}\left\|z_{n}-q\right\| \cdot\left\|x_{n+1}-q\right\|+\gamma_{n}\left\|x_{n}-q\right\| \cdot\left\|x_{n+1}-q\right\| \\
\leq & \left(1-\alpha_{n}(1-k)\right)\left\|x_{n}-q\right\| \cdot\left\|x_{n+1}-q\right\|+\alpha_{n}\left\langle f(q)-q, x_{n+1}-q\right\rangle \\
\leq & \frac{1}{2}\left(1-\alpha_{n}(1-k)\right)\left(\left\|x_{n}-q\right\|^{2}+\left\|x_{n+1}-q\right\|^{2}\right) \\
& +\alpha_{n}\left\langle f(q)-q, x_{n+1}-q\right\rangle,
\end{aligned}
$$

it turns out that

$$
\left\|x_{n+1}-q\right\|^{2} \leq\left(1-\alpha_{n}(1-k)\right)\left\|x_{n}-q\right\|^{2}+2 \alpha_{n}\left\langle f(q)-q, x_{n+1}-q\right\rangle .
$$

By use of condition (iv), we obtain from Lemma 2.9 that

$$
\lim _{n \rightarrow \infty}\left\|x_{n}-q\right\|^{2}=0
$$

i.e., $x_{n} \rightarrow q$ as $n \rightarrow \infty$.

It is easy to see that the variational inequality (3.2) can be rewritten as

$$
\langle f(q)-q, q-x\rangle \geq 0, \quad \forall x \in \Omega
$$

From Lemma 2.1, it is equivalent to the following fixed point equation:

$$
P_{\Omega} f(q)=q
$$

This completes the proof. 


\section{Theorems}

Theorem 4.1 Let $H$ be a real Hilbert space, and let $C$ be a nonempty closed convex subset of $H$. Let $F: C \times C \rightarrow \mathbb{R}$ be a bifunction which satisfies (A1)-(A4), and let $f: C \rightarrow C$ be a $k$-contraction with the constant $k \in(0,1)$. Let $T: C \rightarrow H$ be a $\tau$-ism mapping with $\tau>0$. Let $\left\{S_{i}: C \rightarrow C\right\}$ be a family of infinitely nonexpansive mappings. Assume that $\Omega=\operatorname{GEP}(F, T) \cap$ $\bigcap_{i=1}^{\infty} F\left(S_{i}\right) \neq \emptyset$. Let $\left\{\alpha_{n}\right\},\left\{\beta_{n}\right\}$ and $\left\{\gamma_{n}\right\}$ be sequences in $(0,1)$ such that $\alpha_{n}+\beta_{n}+\gamma_{n}=1$. Let $\left\{\lambda_{n}\right\}$ be a positive number sequence. Let $x_{1} \in C$ and let $\left\{x_{n}\right\}$ be a sequence generated by

$$
x_{n+1}=\alpha_{n} f\left(y_{n}\right)+\beta_{n} W_{n} u_{n}+\gamma_{n} x_{n}, \quad \forall n \in \mathbb{N}
$$

where $\left\{u_{n}\right\}$ is such that

$$
F\left(u_{n}, y\right)+\left\langle T x_{n}, y-u_{n}\right\rangle+\frac{1}{\lambda_{n}}\left\langle y-u_{n}, u_{n}-x_{n}\right\rangle \geq 0, \quad \forall y \in C,
$$

and $\left\{W_{n}\right\}$ is the sequence generated in (2.1). Assume that the following conditions hold:

(1) $0<c \leq \lambda_{n} \leq d<2 \tau$ and $\lim _{n \rightarrow \infty}\left|\lambda_{n+1}-\lambda_{n}\right|=0$;

(2) $\lim _{n \rightarrow \infty} \alpha_{n}=0, \sum_{n=1}^{\infty} \alpha_{n}=\infty$;

(3) $0<\liminf _{n \rightarrow \infty} \gamma_{n} \leq \limsup _{n \rightarrow \infty} \gamma_{n}<1$,

where $c$ and $d$ are real constants. Then $\left\{x_{n}\right\}$ converges strongly to a point $q \in \Omega$, which solves uniquely the variational inequality (3.2).

Theorem 4.2 Let $H$ be a real Hilbert space, and let $C$ be a nonempty closed convex subset of $H$. Let $F: C \times C \rightarrow \mathbb{R}$ be a bifunction which satisfies (A1)-(A4), and let $f: C \rightarrow C$ be a $k$-contraction with the constant $k \in(0,1)$. Let $A: C \rightarrow H$ be an $\alpha$-ism mapping with $\alpha>0$. Let $T: C \rightarrow H$ be a $\tau$-ism mapping with $\tau>0$. Let $N$ be a maximal monotone operator on $H$ such that the domain of $N$ is included in C. Let $J_{r}=(I+r N)^{-1}$ be the resolvent of $N$ for $r>0$. Let $\left\{S_{i}: C \rightarrow C\right\}$ be a family of infinitely nonexpansive mappings. Assume that $\Omega=\operatorname{GEP}(F, T) \cap \bigcap_{i=1}^{\infty} F\left(S_{i}\right) \cap(A+N)^{-1} 0 \neq \emptyset$. Let $\left\{\alpha_{n}\right\},\left\{\beta_{n}\right\}$ and $\left\{\gamma_{n}\right\}$ be sequences in $(0,1)$ such that $\alpha_{n}+\beta_{n}+\gamma_{n}=1$. Let $\left\{r_{n}\right\}$ and $\left\{\lambda_{n}\right\}$ be positive number sequences. Let $x_{1} \in C$ and let $\left\{x_{n}\right\}$ be a sequence generated by

$$
\left\{\begin{array}{l}
y_{n}=J_{r_{n}}\left(u_{n}-r_{n} A u_{n}\right) \\
x_{n+1}=\alpha_{n} f\left(y_{n}\right)+\beta_{n} W_{n} y_{n}+\gamma_{n} x_{n}, \quad \forall n \in \mathbb{N}
\end{array}\right.
$$

where $\left\{u_{n}\right\}$ is such that

$$
F\left(u_{n}, y\right)+\left\langle T x_{n}, y-u_{n}\right\rangle+\frac{1}{\lambda_{n}}\left\langle y-u_{n}, u_{n}-x_{n}\right\rangle \geq 0, \quad \forall y \in C,
$$

and $\left\{W_{n}\right\}$ is the sequence generated in (2.1). Assume that the following conditions hold:

(1) $0<a \leq r_{n} \leq b<2 \alpha$ and $\lim _{n \rightarrow \infty}\left|r_{n+1}-r_{n}\right|=0$;

(2) $0<c \leq \lambda_{n} \leq d<2 \tau$ and $\lim _{n \rightarrow \infty}\left|\lambda_{n+1}-\lambda_{n}\right|=0$;

(3) $\lim _{n \rightarrow \infty} \alpha_{n}=0, \sum_{n=1}^{\infty} \alpha_{n}=\infty$;

(4) $0<\liminf _{n \rightarrow \infty} \gamma_{n} \leq \limsup _{n \rightarrow \infty} \gamma_{n}<1$,

where $a, b, c$, and $d$ are real constants. Then $\left\{x_{n}\right\}$ converges strongly to a point $q \in \Omega$, which solves uniquely the variational inequality (3.2). 
Theorem 4.3 Let $H$ be a real Hilbert space, and let $C$ be a nonempty closed convex subset of $H$. Let $F: C \times C \rightarrow \mathbb{R}$ be a bifunction which satisfies (A1)-(A4), and let $f: C \rightarrow C$ be a $k$-contraction with the constant $k \in(0,1)$. Let $A: C \rightarrow H$ be an $\alpha$-ism mapping with $\alpha>0$, let $B: C \rightarrow H$ be a $\beta$-ism mapping with $\beta>0$, and let $T: C \rightarrow H$ be a $\tau$-ism mapping with $\tau>0$. Let $\left\{S_{i}: C \rightarrow C\right\}$ be a family of infinitely nonexpansive mappings. Assume that $\Omega=\operatorname{GEP}(F, T) \cap \bigcap_{i=1}^{\infty} F\left(S_{i}\right) \cap \operatorname{VI}(C, A) \cap \operatorname{VI}(C, B) \neq \emptyset$. Let $\left\{\alpha_{n}\right\},\left\{\beta_{n}\right\}$ and $\left\{\gamma_{n}\right\}$ be sequences in $(0,1)$ such that $\alpha_{n}+\beta_{n}+\gamma_{n}=1$. Let $\left\{r_{n}\right\},\left\{\lambda_{n}\right\}$ and $\left\{s_{n}\right\}$ be positive number sequences. Let $x_{1} \in C$ and let $\left\{x_{n}\right\}$ be a sequence generated by

$$
\left\{\begin{array}{l}
y_{n}=P_{C}\left(u_{n}-r_{n} A u_{n}\right), \\
x_{n+1}=\alpha_{n} f\left(y_{n}\right)+\beta_{n} W_{n} P_{C}\left(y_{n}-s_{n} B y_{n}\right)+\gamma_{n} x_{n}, \quad \forall n \in \mathbb{N},
\end{array}\right.
$$

where $\left\{u_{n}\right\}$ is such that

$$
F\left(u_{n}, y\right)+\left\langle T x_{n}, y-u_{n}\right\rangle+\frac{1}{\lambda_{n}}\left\langle y-u_{n}, u_{n}-x_{n}\right\rangle \geq 0, \quad \forall y \in C,
$$

and $\left\{W_{n}\right\}$ is the sequence generated in (2.1). Assume that the following conditions hold:

(1) $0<a \leq r_{n} \leq b<2 \alpha$ and $\lim _{n \rightarrow \infty}\left|r_{n+1}-r_{n}\right|=0$;

(2) $0<c \leq \lambda_{n} \leq d<2 \tau$ and $\lim _{n \rightarrow \infty}\left|\lambda_{n+1}-\lambda_{n}\right|=0$;

(3) $0<e \leq s_{n} \leq g<2 \beta$ and $\lim _{n \rightarrow \infty}\left|s_{n+1}-s_{n}\right|=0$;

(4) $\lim _{n \rightarrow \infty} \alpha_{n}=0, \sum_{n=1}^{\infty} \alpha_{n}=\infty$;

(5) $0<\liminf _{n \rightarrow \infty} \gamma_{n} \leq \limsup _{n \rightarrow \infty} \gamma_{n}<1$,

where $a, b, c, d, e$, and $g$ are real constants. Then $\left\{x_{n}\right\}$ converges strongly to a point $q \in \Omega$, which solves uniquely the variational inequality (3.2).

Proof Put $N=\partial i_{C}$ and $M=\partial i_{C}$ in Theorem 3.1. Then, for $r_{n}>0$ and $s_{n}>0$, we have that $J_{r_{n}}=P_{C}$ and $J_{s_{n}}=P_{C}$. Furthermore, we have $\left(A+\partial i_{C}\right)^{-1} 0=\mathrm{VI}(C, A)$ and $\left(B+\partial i_{C}\right)^{-1} 0=$ $\mathrm{VI}(C, B)$. Indeed, for $q \in C$, we have

$$
\begin{aligned}
q \in\left(A+\partial i_{C}\right)^{-1} 0 & \Longleftrightarrow 0 \in A q+\partial i_{C}(q) \\
& \Longleftrightarrow 0 \in A q+N_{C} q \\
& \Longleftrightarrow-A q \in N_{C} q \\
& \Longleftrightarrow\langle-A q, p-q\rangle \leq 0, \quad \forall p \in C \\
& \Longleftrightarrow\langle A q, p-q\rangle \geq 0, \quad \forall p \in C \\
& \Longleftrightarrow q \in \operatorname{VI}(C, A) .
\end{aligned}
$$

Similarly, for $q \in C$, we also have

$$
q \in\left(B+\partial i_{C}\right)^{-1} 0 \quad \Longleftrightarrow q \in \mathrm{VI}(C, B)
$$

Thus, we obtain the desired result by Theorem 3.1.

Theorem 4.4 Let $H$ be a real Hilbert space, and let $C$ be a nonempty closed convex subset of $H$. Let $F: C \times C \rightarrow \mathbb{R}$ be a bifunction which satisfies (A1)-(A4), and let $f: C \rightarrow C$ be a 
$k$-contraction with the constant $k \in(0,1)$. Let $A: C \rightarrow H$ be an $\alpha$-ism mapping with $\alpha>0$, and let $B: C \rightarrow H$ be a $\beta$-ism mapping with $\beta>0$. Let $\left\{S_{i}: C \rightarrow C\right\}$ be a family of infinitely nonexpansive mappings. Assume that $\Omega=\mathrm{EP}(F) \cap \bigcap_{i=1}^{\infty} F\left(S_{i}\right) \cap \mathrm{VI}(C, A) \cap \mathrm{VI}(C, B) \neq \emptyset$. Let $\left\{\alpha_{n}\right\},\left\{\beta_{n}\right\}$ and $\left\{\gamma_{n}\right\}$ be sequences in $(0,1)$ such that $\alpha_{n}+\beta_{n}+\gamma_{n}=1$. Let $\left\{r_{n}\right\},\left\{\lambda_{n}\right\}$ and $\left\{s_{n}\right\}$ be positive number sequences. Let $x_{1} \in C$ and let $\left\{x_{n}\right\}$ be a sequence generated by

$$
\left\{\begin{array}{l}
y_{n}=P_{C}\left(u_{n}-r_{n} A u_{n}\right), \\
x_{n+1}=\alpha_{n} f\left(y_{n}\right)+\beta_{n} W_{n} P_{C}\left(y_{n}-s_{n} B y_{n}\right)+\gamma_{n} x_{n}, \quad \forall n \in \mathbb{N},
\end{array}\right.
$$

where $\left\{u_{n}\right\}$ is such that

$$
F\left(u_{n}, y\right)+\frac{1}{\lambda_{n}}\left\langle y-u_{n}, u_{n}-x_{n}\right\rangle \geq 0, \quad \forall y \in C,
$$

and $\left\{W_{n}\right\}$ is the sequence generated in (2.1). Assume that the following conditions hold:

(1) $0<a \leq r_{n} \leq b<2 \alpha$ and $\lim _{n \rightarrow \infty}\left|r_{n+1}-r_{n}\right|=0$;

(2) $0<c \leq \lambda_{n} \leq d<2 \tau$ and $\lim _{n \rightarrow \infty}\left|\lambda_{n+1}-\lambda_{n}\right|=0$;

(3) $0<e \leq s_{n} \leq g<2 \beta$ and $\lim _{n \rightarrow \infty}\left|s_{n+1}-s_{n}\right|=0$;

(4) $\lim _{n \rightarrow \infty} \alpha_{n}=0, \sum_{n=1}^{\infty} \alpha_{n}=\infty$;

(5) $0<\liminf _{n \rightarrow \infty} \gamma_{n} \leq \limsup _{n \rightarrow \infty} \gamma_{n}<1$,

where $a, b, c, d$, e, and $g$ are real constants. Then $\left\{x_{n}\right\}$ converges strongly to a point $q \in \Omega$, which solves uniquely the variational inequality (3.2).

Proof In Theorem 4.3, put $T=0$. Then, for all $\tau \in(0, \infty)$, we have

$$
\langle x-y, T x-T y\rangle \geq \tau\|T x-T y\|^{2}, \quad \forall x, y \in C .
$$

Taking $c, d \in(0, \infty)$ with $0<c<d<\infty$ and choosing a sequence $\left\{\lambda_{n}\right\}$ of real numbers with $c \leq \lambda_{n} \leq d$, we obtain the desired result by Theorem 4.3 .

Theorem 4.5 Let $H$ be a real Hilbert space, and let $C$ be a nonempty closed convex subset of $H$. Let $P_{C}: H \rightarrow C$ be the metric projection. Let $f: C \rightarrow C$ be a k-contraction with the constant $k \in(0,1)$. Let $A: C \rightarrow H$ be an $\alpha$-ism mapping with $\alpha>0$, let $B: C \rightarrow H$ be a $\beta$-ism mapping with $\beta>0$, and let $T: C \rightarrow H$ be a $\tau$-ism mapping with $\tau>0$. Let $\left\{S_{i}: C \rightarrow C\right\}$ be a family of infinitely nonexpansive mappings. Assume that $\Omega=\operatorname{VI}(C, T) \cap$ $\bigcap_{i=1}^{\infty} F\left(S_{i}\right) \cap \operatorname{VI}(C, A) \cap \operatorname{VI}(C, B) \neq \emptyset$. Let $\left\{\alpha_{n}\right\},\left\{\beta_{n}\right\}$ and $\left\{\gamma_{n}\right\}$ be sequences in $(0,1)$ such that $\alpha_{n}+\beta_{n}+\gamma_{n}=1$. Let $\left\{r_{n}\right\},\left\{\lambda_{n}\right\}$ and $\left\{s_{n}\right\}$ be positive number sequences. Let $x_{1} \in C$ and let $\left\{x_{n}\right\}$ be a sequence generated by

$$
\left\{\begin{array}{l}
u_{n}=P_{C}\left(x_{n}-\lambda_{n} T x_{n}\right), \\
y_{n}=P_{C}\left(u_{n}-r_{n} A u_{n}\right), \\
x_{n+1}=\alpha_{n} f\left(y_{n}\right)+\beta_{n} W_{n} P_{C}\left(y_{n}-s_{n} B y_{n}\right)+\gamma_{n} x_{n}, \quad \forall n \in \mathbb{N},
\end{array}\right.
$$

where $\left\{W_{n}\right\}$ is the sequence generated in (2.1). $\left\{\lambda_{n}\right\},\left\{s_{n}\right\},\left\{r_{n}\right\},\left\{\alpha_{n}\right\}$ and $\left\{\gamma_{n}\right\}$ satisfy the following conditions:

(1) $0<a \leq r_{n} \leq b<2 \alpha$ and $\lim _{n \rightarrow \infty}\left|r_{n+1}-r_{n}\right|=0$;

(2) $0<c \leq \lambda_{n} \leq d<2 \tau$ and $\lim _{n \rightarrow \infty}\left|\lambda_{n+1}-\lambda_{n}\right|=0$; 
(3) $0<e \leq s_{n} \leq g<2 \beta$ and $\lim _{n \rightarrow \infty}\left|s_{n+1}-s_{n}\right|=0$;

(4) $\lim _{n \rightarrow \infty} \alpha_{n}=0, \sum_{n=1}^{\infty} \alpha_{n}=\infty$;

(5) $0<\liminf _{n \rightarrow \infty} \gamma_{n} \leq \limsup _{n \rightarrow \infty} \gamma_{n}<1$,

where $a, b, c, d, e$, and $g$ are real constants. Then $\left\{x_{n}\right\}$ converges strongly to a point $q \in \Omega$, which solves uniquely the variational inequality (3.2).

Proof In Theorem 4.3, put $F=0$. Then we find that

$$
\left\langle T x_{n}, y-u_{n}\right\rangle+\frac{1}{\lambda_{n}}\left\langle y-u_{n}, u_{n}-x_{n}\right\rangle \geq 0, \quad \forall y \in C
$$

is equivalent to

$$
\left\langle y-u_{n}, x_{n}-\lambda_{n} T x_{n}-u_{n}\right\rangle \leq 0, \quad \forall y \in C
$$

i.e., by Lemma 2.1, $u_{n}=P_{C}\left(x_{n}-\lambda_{n} T x_{n}\right)$. This completes the proof.

Theorem 4.6 Let $H$ be a real Hilbert space, and let $C$ be a nonempty closed convex subset of $H$. Let $P_{C}: H \rightarrow C$ be the metric projection. Let $f: C \rightarrow C$ be a $k$-contraction with the constant $k \in(0,1)$. Let $A: C \rightarrow H$ be an $\alpha$-ism mapping with $\alpha>0$, let $B: C \rightarrow H$ be a $\beta$-ism mapping with $\beta>0$, and let $T: C \rightarrow H$ be a $\tau$-ism mapping with $\tau>0$. Let $\left\{S_{i}\right.$ : $C \rightarrow C\}$ be a family of infinitely nonexpansive mappings. Let $Y: C \rightarrow H$ be a widely $r$-strict pseudo-contraction with $r<1(r \in \mathbb{R}) . Z: C \rightarrow H$ be a widely s-strict pseudo-contraction with $s<1(s \in \mathbb{R})$. Assume that $\Omega=\operatorname{VI}(C, T) \cap \bigcap_{i=1}^{\infty} F\left(S_{i}\right) \cap \operatorname{Fix}(Y) \cap \operatorname{Fix}(Z) \neq \emptyset$. Let $\left\{\alpha_{n}\right\}$, $\left\{\beta_{n}\right\}$ and $\left\{\gamma_{n}\right\}$ be sequences in $(0,1)$ such that $\alpha_{n}+\beta_{n}+\gamma_{n}=1$. Let $\left\{r_{n}\right\},\left\{\lambda_{n}\right\}$ and $\left\{s_{n}\right\}$ be positive number sequences. Let $x_{1} \in C$ and let $\left\{x_{n}\right\}$ be a sequence generated by

$$
\left\{\begin{array}{l}
u_{n}=P_{C}\left(x_{n}-\lambda_{n} T x_{n}\right), \\
y_{n}=P_{C}\left(u_{n}-r_{n} A u_{n}\right), \\
x_{n+1}=\alpha_{n} f\left(y_{n}\right)+\beta_{n} W_{n} P_{C}\left(y_{n}-s_{n} B y_{n}\right)+\gamma_{n} x_{n}, \quad \forall n \in \mathbb{N}
\end{array}\right.
$$

where $\left\{W_{n}\right\}$ is the sequence generated in (2.1). $\left\{\lambda_{n}\right\},\left\{s_{n}\right\},\left\{r_{n}\right\},\left\{\alpha_{n}\right\}$ and $\left\{\gamma_{n}\right\}$ satisfy conditions (i)-(v) respectively, which appear in Theorem 3.1. $\left\{t_{n}\right\}$ satisfies

(1) $\left\{t_{n}\right\} \subset(-\infty, 1)$;

(2) $r \leq t_{n} \leq l<1$

(3) $\sum_{n=1}^{\infty}\left|t_{n}-t_{n+1}\right|<\infty$.

Then $\left\{x_{n}\right\}$ converges strongly to a point $q_{0} \in \Omega$.

Proof Put $N=\partial i_{C}$ and $A=I-Y$ in Theorem 3.1. Furthermore, put $p=1-l, r_{n}=1-t_{n}$ and $2 \alpha=1-r$ in Theorem 3.1. From $\left\{t_{n}\right\} \subset(-\infty, 1)$ and $r \leq t_{n} \leq l<1$, we get $\left\{r_{n}\right\} \subset(0, \infty)$ and $0<p \leq r_{n} \leq 2 \alpha$. We also get

$$
\sum_{n=1}^{\infty}\left|r_{n+1}-r_{n}\right|=\sum_{n=1}^{\infty}\left|t_{n+1}-t_{n}\right|<\infty
$$

and

$$
I-r_{n} A=I-\left(1-t_{n}\right)(I-Y)=\left(1-t_{n}\right) Y+t_{n} I .
$$


Furthermore, we have $\left(A+\partial i_{C}\right)^{-1} 0=\operatorname{Fix}(Y)$. Indeed, for $q \in C$, we have

$$
\begin{aligned}
q \in\left(A+\partial i_{C}\right)^{-1} 0 & \Longleftrightarrow 0 \in A q+\partial i_{C}(q) \\
& \Longleftrightarrow 0 \in q-Y q+N_{C}(q) \\
& \Longleftrightarrow Y q-q \in N_{C}(q) \\
& \Longleftrightarrow\langle Y q-q, p-q\rangle \leq 0, \quad \forall p \in C \\
& \Longleftrightarrow P_{C} Y(q)=q .
\end{aligned}
$$

Since $\operatorname{Fix}(Y) \neq \emptyset$, we get from [27] that $\operatorname{Fix}\left(P_{C} Y\right)=\operatorname{Fix}(Y)$. Thus we obtain the desired result by Theorem 3.1.

Similarly, put $M=\partial i_{C}$ and $B=I-Z$ in Theorem 3.1. Furthermore, put $p=1-l, s_{n}=1-t_{n}$ and $2 \beta=1-s$ in Theorem 3.1. From $\left\{t_{n}\right\} \subset(-\infty, 1)$ and $s \leq t_{n} \leq l<1$, we get $\left\{s_{n}\right\} \subset(0, \infty)$ and $0<p \leq s_{n} \leq 2 \beta$. We also get

$$
\sum_{n=1}^{\infty}\left|s_{n+1}-s_{n}\right|=\sum_{n=1}^{\infty}\left|t_{n+1}-t_{n}\right|<\infty
$$

and

$$
I-s_{n} B=I-\left(1-t_{n}\right)(I-Z)=\left(1-t_{n}\right) Z+t_{n} I .
$$

Furthermore, we also obtain $\left(B+\partial i_{C}\right)^{-1} 0=\operatorname{Fix}(Z)$.

Due to Section 3 and Section 4, we will give our conclusion in the next section.

\section{Conclusion}

Methods for solving a generalized equilibrium problem, a fixed point problem and the zero points of the sum of two operators have been studied by many authors respectively. However, in this paper, for finding a common solution of the above three problems, we proposed a new regularization algorithm, and it is proved that the sequence generated by this algorithm has the strong convergence. And then some corollaries to this strong convergence theorem are presented, which play important roles in nonlinear analysis and optimization problem.

\section{Competing interests}

The authors declare that they have no competing interests.

\section{Authors' contributions}

All the authors read and approved the final manuscript.

\section{Acknowledgements}

The authors thank the referees for their helpful comments, which improved the presentation of this paper. This work was supported by the Foundation of Tianjin Key Laboratory for Advanced Signal Processing.

Received: 14 July 2015 Accepted: 23 September 2015 Published online: 06 October 2015

\section{References}

1. Browder, FE: Nonlinear operators and nonlinear equations of evolution in Banach spaces. Proc. Symp. Pure Math. 18, 78-81 (1967) 
2. Blum, E, Oetti, W: From optimization and variational inequalities to equilibrium problems. Math. Stud. 63, 123-145 (1994)

3. Kim, KS, Kim, JK, Lim, WH: Convergence theorems for common solutions of various problems with nonlinear mapping. J. Inequal. Appl. 2014, 2 (2014)

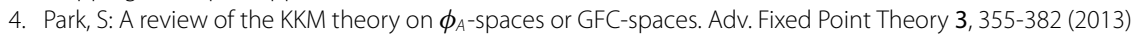

5. Zegeye, $\mathrm{H}$, Shahzad, $\mathrm{N}$ : Strong convergence theorem for a common point of solution of variational inequality and fixed point problem. Adv. Fixed Point Theory 2, 374-397 (2012)

6. Rockafellar, RT: On the maximality of sums of nonlinear monotone operators. Trans. Am. Math. Soc. 149, 75-88 (1970)

7. Chang, SS, Lee, HWJ, Chan, CK: A new method for solving equilibrium problem fixed point problem and variational inequality problem with application to optimization. Nonlinear Anal. 70, 3307-3319 (2009)

8. Takahashi, S, Takahashi, W: Viscosity approximation methods for equilibrium problems and fixed point problems in Hilbert spaces. J. Math. Anal. Appl. 331, 506-515 (2007)

9. Jeong, JU: Strong convergence theorems for a generalized mixed equilibrium problem and variational inequality problems. Fixed Point Theory Appl. 2013, 65 (2013)

10. Rodjanadid, B, Sompong, S: A new iterative method for solving a system of generalized equilibrium problems, generalized mixed equilibrium problems and common fixed point problem in Hilbert spaces. Adv. Fixed Point Theory 3, 675-705 (2013)

11. Cho, SY: Iterative processes for common fixed point of two different families of mappings with applications. J. Glob. Optim. 57, 1429-1446 (2013)

12. Cho, SY, Qin, X: On the strong convergence of an iterative process for asymptotically strict pseudocontractions and equilibrium problems. Appl. Math. Comput. 235, 430-438 (2014)

13. Li, DF, Zhao, J: On variational inequality, fixed point and generalized mixed equilibrium problems. J. Inequal. Appl. 2014, 203 (2014)

14. Shimoji, K, Takahashi, W: Strong convergence to common fixed points of infinite nonexpansive mappings and applications. Taiwan. J. Math. 5, 387-404 (2001)

15. Rockafellar, RT: On the maximal monotonicity of subdifferential mappings. Pac. J. Math. 33, 209-216 (1970)

16. Hao, Y: On variational inclusion and common fixed point problems in Hilbert spaces with applications. Appl. Math. Comput. 217, 3000-3010 (2010)

17. Yuan, Q, Zhang, YP: Convergence of a regularization algorithm for nonexpansive and monotone operators in Hilbert spaces. Fixed Point Theory Appl. 2014, 180 (2014)

18. Takahashi, W: Introduction to Nonlinear and Convex Analysis. Yokohama Publishers, Yokohama (2009)

19. Xu, HK: Viscosity approximation methods for nonexpansive mappings. J. Math. Anal. Appl. 298, 279-291 (2004)

20. Eshita, K, Takahashi, W: Approximating zero points of accretive operators in general Banach spaces. JP J. Fixed Point Theory Appl. 2, 105-116 (2007)

21. Takahashi, S, Takahashi, W, Toyoda, M: Strong convergence theorems for maximal monotone operators with nonlinear mappings in Hilbert spaces. J. Optim. Theory Appl. 147, 27-41 (2010)

22. Takahashi, W: Nonlinear Functional Analysis. Yokohama Publishers, Yokohama (2000)

23. Suzuki, T: Strong convergence of Krasnoselskii and Mann's type sequences for one-parameter nonexpansive semigroups without Bochner integrals. J. Math. Anal. Appl. 305, 227-239 (2005)

24. Hundal, H: An alternating projection that does not converge in norm. Nonlinear Anal. 57, $35-61$ (2004)

25. Xu, HK: Averaged mappings and the gradient-projection algorithm. J. Optim. Theory Appl. 150, 360-378 (2011)

26. Aoyama, K, Kimura, Y, Takahashi, W, Toyoda, M: On a strongly nonexpansive sequence in Hilbert spaces. J. Nonlinear Convex Anal. 8, 471-489 (2007)

27. Zhou, $\mathrm{H}$ : Convergence theorems of fixed points for $\mathrm{k}$-strict pseudo-contractions in Hilbert spaces. Nonlinear Anal. 69 , 456-462 (2008)

\section{Submit your manuscript to a SpringerOpen ${ }^{\circ}$ journal and benefit from:}

- Convenient online submission

Rigorous peer review

- Immediate publication on acceptance

- Open access: articles freely available online

- High visibility within the field

- Retaining the copyright to your article 